\title{
Validation of an Approximate REML Algorithm for Parameter Estimation in a Multitrait, Multiple Across-Country Evaluation Model: A Simulation Study
}

\author{
J. Tarrés, ${ }^{*} \dagger^{1}$ Z. Liu, ${ }^{*}$ V. Ducrocq, $†$ F. Reinhardt, ${ }^{*}$ and R. Reents ${ }^{*}$ \\ *Vereinigte Informationssysteme Tierhaltung w.v., Heideweg 1, 27283 Verden, Germany \\ †UR337, Station de Génétique Quantitative et Appliquée, Institut National de la Recherche Agronomique, 78352 Jouy-en-Josas Cedex, France
}

\section{ABSTRACT}

A multitrait, multiple across-country evaluation (MT-MACE) model permitting a variable number of correlated traits per country allows international genetic evaluation models to more closely match national models. Before the MT-MACE evaluation can be applied, genetic (co)variance components within and across country must be estimated. An approximate REML algorithm for parameter estimation was developed and was validated via simulation. This method is based on the expectation maximization REML (EMREML) algorithm. Because obtaining the inverse of coefficient matrix is not usually feasible for large amounts of data, an algorithm using the multiple-trait effective daughter contribution (EDC) is proposed to provide approximate diagonal elements of the inverse matrix. The accuracy of the approximate EM-REML was tested with simulated data and compared with an average information REML (AI-REML) from available software. Two simulation studies were performed. First, data of 2 countries were simulated using a single-trait model. Estimates of across-country genetic correlations with the developed algorithm were unbiased and very precise. The precision, however, depended on the percentage of bulls with data in both countries. The results obtained with the approximate EM-REML software were very close to those obtained with the AI-REML software regarding estimated genetic correlations and bulls' estimated breeding values. The second simulation assumed a multiple trait model and the same number of traits, pedigree structure, EDC, and pattern of missing records as for actual observations for milk yield obtained from French and German national Holstein evaluations. As with the single-trait scenarios, the approximate EM-REML gave nearly unbiased and very precise estimates of within- and across-country genetic correla-

Received February 2, 2007.

Accepted June 11, 2007.

${ }^{1}$ Corresponding author: joaquim.tarres@dga.jouy.inra.fr tions. The results obtained in both simulation studies confirmed the suitability of the MT-MACE model and approximate EM-REML software in a wide range of situations. Even when the genetic trend was incorrectly estimated by the national evaluations, a joint analysis including a time effect in the MT-MACE model adequately corrected for this bias.

Key words: multiple across-country evaluation, restricted maximum likelihood, daughter yield deviation, effective daughter contribution

\section{INTRODUCTION}

The multiple across-country evaluation (MACE; Schaeffer, 1994) methodology is used for international dairy bull comparisons. Estimated breeding values in national genetic evaluations are deregressed within each country to obtain the values of the dependent variable for bulls that have daughters with records. Currently, a single EBV per bull is permitted for each country in international genetic evaluations by Interbull. As more and more countries have upgraded their national genetic evaluation system to a multiple-trait model or a multiple-lactation random regression test-day model (RRTDM), differences among models for national and international evaluations have become increasingly evident. To optimize genetic evaluation models for both national and international evaluations, Sullivan and Wilton (2001) proposed a multiple-trait MACE (MTMACE) with a variable number of traits per country. This model extended the current single-trait MACE (ST-MACE) to multiple lactations, or traits for countries using a multiple trait model in national genetic evaluation. Schaeffer (2001) developed a multiple trait de-regression method for MACE evaluation. More recently, the simulation study by Sullivan et al. (2005) confirmed the theoretical expectation that MT-MACE methods should be preferred over methods that allow only one trait per country when assuming true genetic parameters. Mark and Sullivan (2006) applied MTMACE to field data for udder health to quantify the benefits in terms of reliability and ability to predict 
international genetic merit of MT-MACE compared with ST-MACE, and to discuss the practical implementation of MT-MACE. They concluded that the MTMACE method is recommended for international genetic udder health evaluations.

In parallel to the development involving de-regression, Ducrocq et al. (2001) suggested using corrected records for a 2-step multitrait genetic evaluation. Indeed, this approach can also be applied to international genetic evaluations. Yield deviations (YD) of cows and daughter yield deviations (DYD) of bulls are, in fact, corrected records. For single-trait models, VanRaden and Wiggans (1991) developed formulas for the calculation of YD and DYD and its daughter equivalent, which were later called effective daughter contribution (EDC). Liu et al. (2004a) extended the YD and DYD derivation to multiple trait models, including RRTDM. Also, Liu et al. (2004a) developed a method for approximating reliabilities of EBV under general multiple trait models, which can also be used to approximate EDC associated with bulls' DYD.

Based on DYD, Liu et al. (2004b) presented an MTMACE model for international bull comparison. This model allowed a variable number of correlated traits per country. For countries using multiple trait models in national genetic evaluations, a vector of DYD and its corresponding EDC matrix are needed for each bull with daughter performance information. An approximate REML algorithm was developed to estimate across country genetic correlations based on multitrait EDC (MTEDC, Liu et al., 2004a). For solving the large equation system of the MT-MACE model, a preconditioned conjugate gradients algorithm (PCG, Strandén and Lidauer, 1999) was applied together with the iteration on data technique. This MACE model can also be used to analyze YD of cows or de-regressed estimates of breeding values.

The aim of this paper was to validate the approximate REML method for estimating across-country genetic correlations via simulation. In addition to the analysis with a ST-MACE model, a MT-MACE analysis was performed to check the suitability of this approximate REML algorithm in a wide range of situations.

\section{MATERIALS AND METHODS}

\section{The MT-MACE Model}

For a country $j$ using a multitrait model in national genetic evaluation, the following statistical model was applied to the data of a bull $i$ from the country $\mathrm{j}$ :

$$
\mathbf{y}_{i j}=\mathbf{f}_{j}+\mathbf{a}_{i j}+\mathbf{e}_{i j}
$$

where $\mathbf{y}_{i j}$ is a vector of data of the $i$ th bull in country $\mathrm{j}$, $\mathbf{f}_{j}$ is a vector of overall means for traits of the $j$ th country, $\mathbf{a}_{i j}$ is a vector of additive genetic effects of bull $i$ in country $j$, and $\mathbf{e}_{i j}$ is a vector of residual effects. Model [1] is also valid for data from countries with a single-trait model in national genetic evaluations, with all terms above becoming scalars. The (co)variance matrix of genetic effects of the $m$ countries and its inverse are denoted as

$$
\begin{gathered}
\mathbf{G}_{0}=\left[\begin{array}{cccc}
\mathbf{G}_{0_{11}} & \mathbf{G}_{0_{12}} & \ldots & \mathbf{G}_{0_{1 m}} \\
& \mathbf{G}_{0_{22}} & \ldots & \mathbf{G}_{0_{2 m}} \\
& & \ddots & \vdots \\
& & & \mathbf{G}_{0_{m m}}
\end{array}\right], \\
\text { and } \mathbf{G}_{0}^{-1}=\left[\begin{array}{ccccc}
\mathbf{G}_{0}^{11} & \mathbf{G}_{0}^{12} & \ldots & \mathbf{G}_{0}^{1 m} \\
& \mathbf{G}_{0}^{22} & \ldots & \mathbf{G}_{0}^{2 m} \\
& & \ddots & \vdots \\
& & & \mathbf{G}_{0}^{m m}
\end{array}\right]
\end{gathered}
$$

where $m$ is the number of countries, $\mathbf{G}_{0_{j j}}$ is the original genetic (co)variance matrix of country $j$, and $\mathbf{G}_{0_{j k}}$ is the genetic covariance matrix between countries $j$ and $k$. When country $j$ uses a single-trait model for national evaluation, $\mathbf{G}_{0_{j j}}$ is a scalar and all corresponding offdiagonal blocks, $\mathbf{G}_{0_{j k}}(j \neq k)$, become vectors or scalars, depending on the number of traits in other countries. As usually considered in MACE, data of a bull from different countries are assumed to be residually uncorrelated. The inverse of error (co)variance matrix of bull $i$ in country $j$ is

$$
\left[\operatorname{Var}\left(\mathbf{e}_{i j}\right)\right]^{-1}=\Psi_{i j}
$$

where $\Psi_{i j}$ is the EDC matrix for bull $i$ in country $j$, converted from the reliability matrix contributed by his daughters' records in the $j$ th country. The MTEDC procedure (Liu et al., 2004a) can be used to approximate matrix $\Psi$ for each bull.

\section{The Mixed Model Equations}

The mixed model equations (MME) of model [1] consist of equations for additive genetic effects of bulls and fixed effects of country means. Ignoring pedigree contributions, the equations corresponding to bull $i$ are 


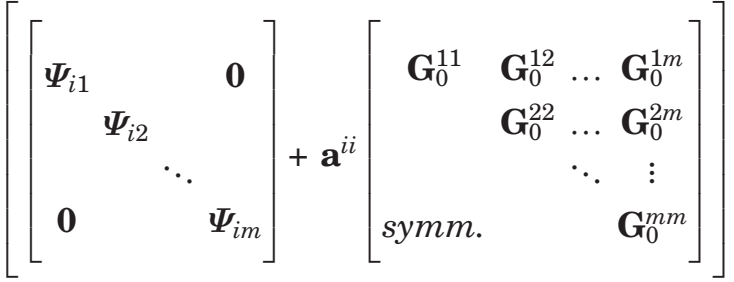

$$
\begin{aligned}
& {\left[\begin{array}{c}
\hat{\mathbf{a}}_{i 1} \\
\hat{\mathbf{a}}_{i 2} \\
\vdots \\
\hat{\mathbf{a}}_{i m}
\end{array}\right]+\left[\begin{array}{llll} 
& & & \\
\boldsymbol{\Psi}_{i 1} & & & \mathbf{0} \\
& \boldsymbol{\Psi}_{i 2} & & \\
& & \ddots & \\
\mathbf{0} & & & \boldsymbol{\Psi}_{i m}
\end{array}\right]\left[\begin{array}{c}
\hat{\mathbf{f}}_{1} \\
\hat{\mathbf{f}}_{2} \\
\ddots \\
\hat{\mathbf{f}}_{m}
\end{array}\right]=\left[\begin{array}{c}
\Delta_{i 1} \\
\Delta_{i 2} \\
\ddots \\
\Delta_{i m}
\end{array}\right]}
\end{aligned}
$$

where $a^{i i}$ is the diagonal element of bull $i$ in the inverse of the numerator relationship matrix $\mathbf{A}$, and $\Delta_{i j}$ represents the right-hand-side (RHS) of bull $i$ in country $j$. Note that $\Delta_{i j}$ is not dependent on across-country correlations and is a function of country-specific information only. The RHS of bull $i$ in country $j$ was calculated as $\Delta_{i j}=\Psi_{i j} \mathbf{y}_{i j}$, multiplying the EDC matrix by the data vector. Equations for the fixed effects of country means in model [1] are

$$
\begin{aligned}
& {\left[\begin{array}{ccccc} 
& & & \\
\boldsymbol{\Psi}_{f 1} & & & \mathbf{0} \\
& \boldsymbol{\Psi}_{f 2} & & \\
& & \ddots & \\
\mathbf{0} & & & \boldsymbol{\Psi}_{f m}
\end{array}\right]\left[\begin{array}{c}
\hat{\mathbf{f}}_{1} \\
\hat{\mathbf{f}}_{2} \\
\vdots \\
\hat{\mathbf{f}}_{m}
\end{array}\right]}
\end{aligned}
$$

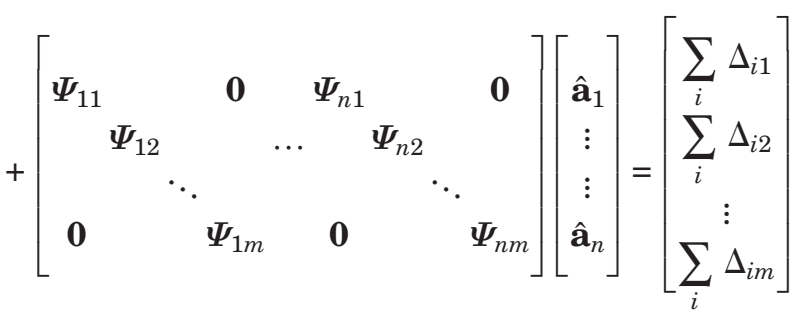

where $n$ is the total number of bulls with DYD, $\hat{\mathbf{a}}_{i}{ }^{\prime}=\left[\begin{array}{llll}\hat{\mathbf{a}}_{i 1}{ }^{\prime} & \hat{\mathbf{a}}_{i 2}{ }^{\prime} \ldots \hat{\mathbf{a}}_{i m}{ }^{\prime}\end{array}\right]$ represents EBV of bull $i$ in all $m$ countries, and $\boldsymbol{\Psi}_{f_{j}}=\sum_{i=1}^{n_{j}} \boldsymbol{\Psi}_{i j}$ with $n_{j}$ being the number of bulls with DYD in country $j$.

The equation system was solved using a PCG algorithm and iteration on data technique (Strandén and Lidauer, 1999; Liu et al., 2004b). The convergence criterion, defined as the base 10 logarithm of the sum of squares of differences in solutions between 2 consecutive rounds of iteration divided by the sum of squares of solutions in last round of iteration, was set to -10 .

\section{Estimation of (Co)Variance Components}

An approximate REML algorithm was proposed by Liu et al. (2004b) to estimate genetic (co)variances components for general MT-MACE models. This algorithm is based on the expectation maximization REML (EMREML) algorithm for the genetic effects only:

$$
\hat{\sigma}_{g_{j k}}^{[l]}=\left[\hat{\mathbf{a}}_{j}^{\prime} \mathbf{A}^{-1} \hat{\mathbf{a}}_{k}+\operatorname{tr}\left(\mathbf{A}^{-1} \mathbf{C}^{j k}\right) / q,\right.
$$

where $\hat{\sigma}_{g_{j k}}^{[l]}$ is the estimated genetic covariance between countries $j$ and $k$ at iteration $l, \hat{\mathbf{a}}_{j}$ is the vector of EBV at iteration $l$ of all animals in country $j$ (animals sorted within trait), $\mathbf{C}^{j k}$ is the submatrix of the inverse of coefficient matrix $(\mathbf{C})$, corresponding to the country pair $j$ and $k$, and $q$ is the total number of animals in the pedigree file. For countries having multiple correlated genetic effects, $j$ and $k$ represent the genetic effects within country.

As the coefficient matrix, $\mathbf{C}$, of MME is impossible to invert for large systems, an alternative formulation of the EM-REML formula is necessary. The numerator relationship matrix can be decomposed as

$$
\mathbf{A}=\mathbf{T D}^{\prime}
$$

where matrix $\mathbf{T}$ is a lower triangular matrix and its inverse $\mathbf{T}^{-1}$ is also a lower triangular matrix with 1 in the diagonals and the only nonzero elements, equal to -0.5 , connect an animal to its known parents; $\mathbf{D}$ is a diagonal matrix for Mendelian sampling variance (Mrode, 2005). As Mendelian sampling effects are $\mathbf{m}_{j}=$ $\mathbf{T}^{-1} \mathbf{a}_{j}$, the EM-REML formula [6] can be reformulated (Fikse et al., 2003) as

$$
\hat{\sigma}_{g_{j k}}^{[l]}=\left[\hat{\mathbf{m}}_{j}{ }^{\prime} \mathbf{D}^{-1} \hat{\mathbf{m}}_{k}+\operatorname{tr}\left(\mathbf{D}^{-1} \operatorname{diag}\left(\mathbf{T}^{-1} \mathbf{C}^{j k}\left(\mathbf{T}^{-1}\right)^{\prime}\right)\right)\right] / q .
$$

The first term of formula [8] is a simple summation of Mendelian sampling estimates over all animals:

$$
\hat{\mathbf{m}}_{j}{ }^{\prime} \mathbf{D}^{-1} \hat{\mathbf{m}}_{k}=\sum_{i=1}^{q} d_{i} \hat{m}_{i j} \hat{m}_{i k}
$$

where $\hat{m}_{i j}$ and $\hat{m}_{i k}$ are the Mendelian sampling estimates of animal $i$ in countries (or within country trait) $j$ and $k$, respectively. For animal $i$, the corresponding diagonal element of the matrix product $\mathbf{T}^{-1} \mathbf{C}^{j k}\left(\mathbf{T}^{-1}\right)^{\prime}$ is

$$
\left\{\operatorname{diag}\left(\mathbf{T}^{-1} \mathbf{C}^{j k}\left(\mathbf{T}^{-1}\right)^{\prime}\right)\right\}_{i}=\mathbf{t}^{\prime} \mathbf{C}_{i}^{j k} \mathbf{t}
$$

where $\mathbf{t}^{\prime}=[1-1 / 2-1 / 2]$, and $\mathbf{C}_{i}^{j k}$ is a submatrix of the inverse of coefficient matrix for country (subtrait) pair 
$j$ and $k$ corresponding to animal $i$, its sire $s$, and its dam $d$. Many of these ideas were reported by VanRaden (2001), also accounting for inbreeding.

From the above formulas [8] and [10], it can be seen that, in addition to diagonal elements of the inverse of the coefficient matrix, off-diagonal elements among animal, sire, and dam are also required for estimating genetic (co)variance components. Because obtaining the inverse of the coefficient matrix is not usually feasible for large-scale equation systems, the MTEDC (Liu et al., 2004a) is used to provide approximate diagonal elements of the inverse matrix. An approximation method is presented here for calculating off-diagonal elements of the inverse matrix corresponding to each animal, sire, and dam trio.

\section{Approximating (Co)Variances of Mendelian Sampling Estimates}

For each animal, the following 2 assumptions were made. First, an animal is the only common progeny of its parents, which is not too restrictive for livestock species such as dairy cattle. Second, there are no environmental covariances between any 2 members of the trio. This assumption is easily met for model [1], which contains only fixed and genetic effects. Under these 2 assumptions, all required elements of the submatrix $\mathbf{C}_{i}^{j k}$ can be obtained from inverting the following matrix for each animal:

$$
\left[\begin{array}{ccc}
\mathbf{\Psi}_{i-s d}^{*}+d_{i} \mathbf{G}_{0}^{-1} & -\frac{1}{2} d_{i} \mathbf{G}_{0}^{-1} & -\frac{1}{2} d_{i} \mathbf{G}_{0}^{-1} \\
& \Psi_{s-1}^{*}+\left(d_{s}+\frac{1}{4} d_{i}\right) \mathbf{G}_{0}^{-1} & \frac{1}{4} d_{i} \mathbf{G}_{0}^{-1} \\
\text { symm. } & & \Psi_{d-i}^{*}+\left(d_{\mathrm{d}}+\frac{1}{4} d_{\mathrm{i}}\right) \mathbf{G}_{0}^{-1}
\end{array}\right]
$$

where $\Psi_{i-s d}^{*}$ is EDC matrix of animal $i$ after taking into account all sources of information, except the parental contribution to the animal; $\Psi_{s-i}^{*}\left(\Psi_{d-i}^{*}\right)$ is the EDC matrix of sire (dam) of animal $i$ after accounting for all sources of information except the contribution of animal $i$ to its sire (dam); and $d_{i}, d_{s}$, and $d_{d}$ are the Mendelian sampling terms respectively for the bull $i$, its sire $s$, and its dam $d$ (Mrode, 2005). The matrix $\mathbf{C}_{i s d}$ is then inverted for animal $i$ :

$$
\mathbf{C}_{i s d}^{-1}=\left[\begin{array}{ccc}
\mathbf{C}_{i}^{i i} & \mathbf{C}_{i}^{i s} & \mathbf{C}_{i}^{i d} \\
& \mathbf{C}_{i}^{s s} & \mathbf{C}_{i}^{s d} \\
s y m m . & & \mathbf{C}_{i}^{d d}
\end{array}\right] .
$$

Note that $\mathbf{C}_{i}^{j k}$ is a $3 \times 3$ matrix and represents a submatrix of $\mathbf{C}_{i s d}^{-1}$. The EDC matrices $\Psi_{i-s d}^{*}, \Psi_{s-i}^{*}$, and
$\Psi_{d-i}^{*}$ are expressed on an animal basis. The MTEDC can be used to efficiently obtain the EDC matrices for all animals.

\section{Procedure for Estimating (Co)Variances Across Countries}

The EM-REML algorithm [8] was combined with the PCG algorithm to estimate (co)variance parameters of the multitrait MACE model [1]. An iterative procedure developed for the parameter estimation comprised 4 steps:

1) Solve the equation system of the MT-MACE model with PCG.

2) Compute the quadratic term for a given (co)variance component using formula [9].

3) Approximate prediction error (co)variances of Mendelian sampling estimates with formulas [11] and [12] and the MTEDC approach (Liu et al., 2004a).

4) Estimate genetic (co)variances using the EMREML formula [8] and derive genetic correlation estimates. Newly estimated (co)variances are used to update covariances for the next iteration.

Repeat steps 1 through 4 until all parameters no longer change at the fourth decimal place. The original country-specific (co)variances can be used as starting values.

\section{SIMULATION OF DATA}

\section{Single-Trait MACE Scenarios}

Records of 5,000 bulls, each having progeny in 2 countries recorded for a single trait, were simulated using the following model:

$$
\mathbf{y}_{i j}=\mathbf{a}_{i j}+\mathbf{e}_{i j}
$$

where $\mathbf{y}_{i j}$ is the simulated DYD vector for bull $i$ in country $j, \mathbf{a}_{i j}$ is a vector of additive genetic effects of bull $i$ in country $j$, and $\mathbf{e}_{i j}$ is a vector of residual effects. True breeding values $\mathbf{a}_{i j}$ of the 5,000 bulls that were progeny of 50 unrelated sires were obtained by adding half the breeding value of their sire $\mathbf{s}_{i j}$ to a value $\mathbf{u}_{i j}$ including the dam contribution and Mendelian sampling; that is, representing three quarters of the total genetic variance. The genetic variances for the 2 countries were arbitrarily chosen to be 4 and 6 , respectively. The genetic correlation between countries was 0.9. Residual values $\mathbf{e}_{i j}$ for the 2 traits were generated accounting for variable amount of information summarized in $\mathbf{y}_{i j}$. Its residual variance was assumed to be heterogeneous: $\operatorname{var}\left(e_{i j}\right)=\sigma_{e, j}^{2} / \omega_{i j}$, where $\sigma_{e, j}^{2}$ is the residual variance for 
country $j$ chosen to result in heritabilities of 0.30 in both countries; and the weight $\omega_{i j}$ was a random number between 1 and 300 and represented approximately an effective number of daughters of bull $i$ in each country $j$. A zero residual correlation was assumed between countries. Two scenarios were studied: one scenario with all bulls having data in both countries $(100 \%$ common bulls) and the other scenario with only $10 \%$ of bulls being common, which represents a typical international bull evaluation with 2 countries involved.

The performance of the approximate REML method was compared using a version of the average information REML (AI-REML) software. Although the latter software was originally not developed for a model such as [13] with heterogeneous residual variances, a simple trick can avoid this limitation (Ducrocq et al., 2001). Let $v_{i j}=\sqrt{\omega_{i j}}$. Multiplying both sides of the model equation [13] by $v_{i j}$, one gets

$$
\mathbf{y}_{i j}^{*}=v_{i j} \mathbf{y}_{i j}=v_{i j} \mathbf{a}_{i j}+\varepsilon_{i j} .
$$

Now, the residual part $\varepsilon_{i j}$ has homogeneous variance: $\operatorname{var}\left(\varepsilon_{i j}\right)=v_{i j}^{2} \operatorname{var}\left(e_{i j}\right)=\sigma_{e, j}^{2}$. The REML estimation of the dispersion parameters of model [14] considering $\mathbf{y}_{i j}^{*}$ as the data and $v_{i j}$ as a continuous covariate gives results identical to the analysis of model [13] (Ducrocq et al., 2001). The version of the AI-REML software from I. Misztal and S. Tsuruta (Misztal et al., 2002) was modified by Druet to impose the constraint that genetic and residual variances are fixed (Druet et al., 2003). This constraint was necessary to allow convergence to be obtained, due to genetic variances quickly going to zero.

\section{Multiple-Trait MACE Scenarios}

Because the previous scenarios were rather simplistic, we further extended the validation to check the performance of the approximate EM-REML method by simulating the actual milk production data from the French and German national evaluations; that is, using the same number of traits, pedigree, EDC, and missing pattern as in a real life situation. Records of animals resembling milk production DYD of bulls included in the Interbull 010 files from French and German national evaluations were simulated using the model in equation [13]. The additive genetic effects were modeled in the same way as in national evaluation. Milk production traits are evaluated in Germany using an RRTDM in which the genetic effects of an animal per lactation are modeled with a normalized orthogonal third-order Legendre polynomial (Liu et al., 2001) and in France with a single repeatability lactation model (Robert-Granié et al., 1999). Thus, bulls with data in Germany had 9 correlated traits; that is, 3 regression coefficients (RRC) for the first 3 lactations; bulls with data in France had data for a single trait.

The February 2006 Interbull bull pedigree file was used to generate true breeding values $\mathbf{a}_{i j}$ of the animals. The complete pedigree file was read from the oldest to the youngest, and the breeding value $\mathbf{a}_{i j}$ was obtained by adding half the breeding values of the sire and dam and the Mendelian sampling term, representing half of the total genetic variance. When the sire or dam was unknown, half of the value of genetic groups plus a value $\mathbf{u}_{i j}$ including the sire or dam contribution (i.e., representing one quarter of the total genetic variance) were added. The values were drawn from a $\operatorname{MVN}(0$, $\mathbf{G}_{0}$ ) distribution, where $\mathbf{G}_{0}$ is the genetic (co)variance matrix. The (co)variance matrix of genetic effects of the two countries and its inverse are defined as:

$$
\mathbf{G}_{0}=\left[\begin{array}{ll}
\mathbf{G}_{0_{11}} & \mathbf{G}_{0_{12}} \\
\mathbf{G}_{0_{12}} & \mathbf{G}_{0_{22}}
\end{array}\right] \text {, and } \mathbf{G}_{0}^{-1}=\left[\begin{array}{ll}
\mathbf{G}_{0}^{11} & \mathbf{G}_{0}^{12} \\
\mathbf{G}_{0}^{12} & \mathbf{G}_{0}^{22}
\end{array}\right] \text {. }
$$

The genetic (co)variance matrix $\mathbf{G}_{0}$ used for simulation is shown in Table 1.

Residual values $\mathbf{e}_{i j}$ were also generated from values drawn from an $\operatorname{MVN}\left(0, \mathbf{R}_{\mathrm{i}}\right)$ distribution, where $\mathbf{R}_{\mathrm{i}}$ is the residual (co)variance matrix which is different for each bull, $i$ :

$$
\mathbf{R}_{i}=\left[\begin{array}{cc}
\mathbf{R}_{i 1} & 0 \\
0 & \mathbf{R}_{i 2}
\end{array}\right] \text {, and } \mathbf{R}_{i}^{-1}=\left[\begin{array}{cc}
\boldsymbol{\Psi}_{i 1} & 0 \\
0 & \boldsymbol{\Psi}_{i 2}
\end{array}\right],
$$

where $\mathbf{R}_{i j}=\operatorname{Var}\left(\mathbf{e}_{i j}\right)=\left[\boldsymbol{\Psi}_{i j}\right]^{-1}$ is the error (co)variance matrix of bull $i$ in country $j$ and it is the inverse of the EDC matrix $\Psi_{i j}$ of bull $i$ in country $j$. As usually assumed in MACE, residuals from daughters of a bull from different countries are uncorrelated. Residual values $\mathbf{e}_{i j}$ were obtained by inverting the EDC matrix from the datasets for parameter estimation. Table 2 has the data and pedigree structures for 2 scenarios of the MT-MACE model, one scenario with full pedigree information in which sires and dams of all bulls were known and the other scenario with reduced pedigree information where all dams of bulls were missing. The data and pedigree structures were the same as those used for parameter estimation of milk production traits from France and Germany with an MT-MACE model (Tarrés et al., 2006). Only bulls born from 1985 to 2001 were included.

\section{Inclusion of a Year Effect}

National genetic evaluation may be biased for several reasons. Examples are lack of information on selection 
Table 1. Simulated values of genetic variances (on the diagonal) and genetic correlations (above the diagonal)

\begin{tabular}{|c|c|c|c|c|c|c|c|c|c|}
\hline \multicolumn{9}{|c|}{ Germany } & \multirow{3}{*}{$\frac{\text { France }}{\text { Single lactation }^{2}}$} \\
\hline \multicolumn{3}{|c|}{$\mathrm{RRC}^{1}$ of first lactation } & \multicolumn{3}{|c|}{ RRC of second lactation } & \multicolumn{3}{|c|}{ RRC of third lactation } & \\
\hline First & Second & Third & First & Second & Third & First & Second & Third & \\
\hline 3.715 & $\begin{array}{l}0.147 \\
0.397\end{array}$ & $\begin{array}{r}-0.479 \\
-0.263 \\
0.184\end{array}$ & $\begin{array}{r}0.835 \\
0.216 \\
-0.444 \\
3.362\end{array}$ & $\begin{array}{r}-0.026 \\
0.697 \\
0.184 \\
0.066 \\
0.801\end{array}$ & $\begin{array}{r}-0.409 \\
-0.295 \\
0.909 \\
-0.435 \\
0.156 \\
0.252\end{array}$ & $\begin{array}{r}0.838 \\
0.193 \\
-0.450 \\
0.970 \\
0.043 \\
-0.435 \\
3.697\end{array}$ & $\begin{array}{r}-0.037 \\
0.642 \\
0.098 \\
0.082 \\
0.961 \\
0.101 \\
0.075 \\
0.849\end{array}$ & $\begin{array}{r}-0.400 \\
-0.271 \\
0.909 \\
-0.429 \\
0.163 \\
0.977 \\
-0.427 \\
0.097 \\
0.293\end{array}$ & $\begin{array}{r}0.900 \\
0.200 \\
-0.400 \\
0.900 \\
0.000 \\
-0.400 \\
0.900 \\
0.000 \\
-0.400 \\
1.000\end{array}$ \\
\hline
\end{tabular}

${ }^{1} \mathrm{RRC}=$ random regression coefficient.

${ }^{2}$ France uses a single repeatability lactation model in national evaluation.

practiced in foreign countries or a single-trait genetic evaluation model without consideration of the selection on correlated traits. To correct such potential biases, Ducrocq et al. (2003) proposed a robust MACE model that contains, in addition to regular effects in MACE model, a fixed effect of time; for example, birth year of animal $\times$ country. To investigate whether the inclusion of a year effect would have a favorable impact on the genetic (co)variance estimates, in this section it is assumed that a trend in Mendelian sampling exists for all traits and that no bulls born from 1985 to 2001 have zero Mendelian sampling expectation. To generate such a bias, the simulation models for all traits were modified to:

$$
\mathbf{y}_{i j}=\delta_{k j}+\mathbf{a}_{i j}+\mathbf{e}_{i j}
$$

An effect, $\delta_{k j}$, equal to $5 \%$ of genetic standard deviation per year was added for each trait in each country $j$. The analysis of such a situation was completed by extending the MME to estimate the year effect and implementing this extension in the approximate REML software for parameter estimation. An objective of including the year effect was to investigate whether parameter estimates would differ from those obtained from the original MT-MACE model without the year effect.

\section{RESULTS}

\section{Single-Trait MACE Scenarios}

The averages of the genetic variance estimates per country and of the across country genetic correlation estimates over 200 replicates were very close to the simulated true values (Table 3 ) for single traits when all bulls had data in both countries (no missing value in the bivariate analysis). Standard deviations of genetic correlation estimates were very small (Table 3). The estimates obtained using the approximate EM-REML algorithm were compared with the ones from an already validated software AI-REML. Although the standard deviation was slightly higher for the approximate EMREML, the estimated genetic correlations, on average, were very similar between both methods. The Pearson correlations of animal EBV obtained from both methods was close to 1 .

Averaged estimates of genetic variances and correlations for the datasets with different percentages of missing bulls were also always close to the simulated, true values (Table 3 ). The estimation procedure seems robust, even with only $10 \%$ of common bulls. The amount of missing bulls slightly increased the standard error of the estimates, although the approximate REML was still very precise (Table 3 ).

Table 2. Data and pedigree information for the multitrait, multiple across-country evaluation model simulation scenarios

\begin{tabular}{|c|c|c|c|c|c|c|}
\hline Pedigree & $\begin{array}{l}\text { Bulls with data } \\
\text { in Germany, } \mathrm{n}\end{array}$ & $\begin{array}{l}\text { Bulls with data } \\
\text { in France, } n\end{array}$ & $\begin{array}{l}\text { Common } \\
\text { bulls, } \mathrm{n}\end{array}$ & $\begin{array}{l}\text { Total bulls } \\
\text { with data, } \mathrm{n}\end{array}$ & $\begin{array}{l}\text { Animals in } \\
\text { pedigree, } \mathrm{n}\end{array}$ & $\begin{array}{l}\text { Phantom } \\
\text { groups, } n\end{array}$ \\
\hline Dams known (full) & 5,168 & 5,060 & 683 & 9,545 & 25,737 & 20 \\
\hline Dams unknown (reduced) & 5,168 & 5,060 & 683 & 9,545 & 10,120 & 2 \\
\hline
\end{tabular}


Table 3. Estimates of genetic variances and correlations between countries under the single-trait, multiple across-country evaluation model using the approximate expectation maximization (EM)-REML and the average information (AI)-REML approach for 2 different percentages of common bulls ${ }^{1}$

\begin{tabular}{|c|c|c|c|c|c|}
\hline & \multirow[b]{2}{*}{ True } & \multicolumn{2}{|c|}{ Approximate EM-REML } & \multicolumn{2}{|c|}{ AI-REML } \\
\hline & & Mean & SD & Mean & $\mathrm{SD}$ \\
\hline \multicolumn{6}{|c|}{ Genetic variance, country 1} \\
\hline All common ${ }^{2}$ & 4 & 3.962 & 0.088 & & \\
\hline $10 \%$ common $^{3}$ & 4 & 3.990 & 0.108 & & \\
\hline \multicolumn{6}{|c|}{ Genetic variance, country 2} \\
\hline All common & 6 & 5.983 & 0.133 & & \\
\hline $10 \%$ common & 6 & 5.981 & 0.156 & & \\
\hline \multicolumn{6}{|c|}{ Genetic correlation } \\
\hline All common & 0.90 & 0.900 & 0.003 & 0.900 & 0.002 \\
\hline $10 \%$ common & 0.90 & 0.901 & 0.008 & 0.902 & 0.008 \\
\hline
\end{tabular}

${ }^{1}$ Mean and standard deviations over 200 replicates.

${ }^{2}$ All bulls have data in both countries.

${ }^{3}$ Only $10 \%$ of bulls have data both countries.

\section{Multiple-Trait MACE Scenarios}

The same genetic (co)variances of milk production traits from the French and German national genetic evaluations were estimated using the simulated data. Means of the German parameters and the across country genetic correlations over 20 replicates (Table 4) were very close to the simulated, true ones (Table 1). Standard deviations of the genetic correlation estimates were smaller for correlations related to the first random regression coefficients (Table 4), because they are more heritable than the correlations of the second and third coefficients. In general, the estimates of genetic correlations were quite precise, even when zero correlations were used as starting values (results not shown).

Although the genetic correlation estimates seemed unbiased, averaged genetic variance estimates over 20 replicates were slightly underestimated (Table 4). This was not the case when datasets were simulated and analyzed with a reduced pedigree file with only animal and sire information but dams being unknown. In this case, means of genetic variance and correlation estimates were also very close to their true values (Table 5). The underestimation might be explained by the higher selection effect in the full pedigree.

\section{Estimation of the Year Effect}

To check the implementation of the estimation of the birth year effect within country for each trait, a simulation was run without generating a birth year effect in the simulated data but including it in the actual analysis. Although there were oscillations over years in the particular replicates, the mean over 200 replicates returned exactly a null slope for the birth year effects over time (Figure 1). The intercept of birth year effects

Table 4. Mean of genetic variances (on the diagonal) and mean of genetic correlations (above the diagonal) under the multitrait, multiple across-country evaluation model estimated using the approximate expectation maximization-REML over 20 replicates with full pedigree information (standard deviations were between 0.002 and 0.022 )

\begin{tabular}{|c|c|c|c|c|c|c|c|c|c|}
\hline \multicolumn{9}{|c|}{ Germany } & \multirow{3}{*}{$\frac{\text { France }}{\text { Single lactation }^{2}}$} \\
\hline \multicolumn{3}{|c|}{$\mathrm{RRC}^{1}$ of first lactation } & \multicolumn{3}{|c|}{ RRC of second lactation } & \multicolumn{3}{|c|}{$\mathrm{RRC}$ of third lactation } & \\
\hline First & Second & Third & First & Second & Third & First & Second & Third & \\
\hline 3.476 & $\begin{array}{l}0.148 \\
0.372\end{array}$ & $\begin{array}{r}-0.482 \\
-0.264 \\
0.171\end{array}$ & $\begin{array}{r}0.836 \\
0.219 \\
-0.446 \\
3.139\end{array}$ & $\begin{array}{r}-0.026 \\
0.696 \\
0.186 \\
0.067 \\
0.749\end{array}$ & $\begin{array}{r}-0.413 \\
-0.294 \\
0.909 \\
-0.439 \\
0.156 \\
0.235\end{array}$ & $\begin{array}{r}0.839 \\
0.198 \\
-0.454 \\
0.970 \\
0.044 \\
-0.441 \\
3.449\end{array}$ & $\begin{array}{r}-0.036 \\
0.642 \\
0.101 \\
0.083 \\
0.962 \\
0.101 \\
0.076 \\
0.798\end{array}$ & $\begin{array}{r}-0.403 \\
-0.270 \\
0.909 \\
-0.432 \\
0.164 \\
0.978 \\
-0.431 \\
0.099 \\
0.273\end{array}$ & $\begin{array}{r}0.900 \\
0.206 \\
-0.405 \\
0.901 \\
0.005 \\
-0.404 \\
0.900 \\
0.005 \\
-0.402 \\
0.941\end{array}$ \\
\hline
\end{tabular}

${ }^{1} \mathrm{RRC}=$ random regression coefficient

${ }^{2}$ France uses a single repeatability lactation model in national evaluation. 
Table 5. Mean of genetic variances (on the diagonal) and mean of genetic correlations (above the diagonal) under the multitrait, multiple across-country evaluation model estimated using the approximate expectation maximization-REML over 20 replicates with reduced pedigree information (all dams being unknown) (standard deviations were between 0.002 and 0.022 )

\begin{tabular}{|c|c|c|c|c|c|c|c|c|c|}
\hline \multicolumn{9}{|c|}{ Germany } & \multirow{3}{*}{$\frac{\text { France }}{\text { Single lactation }^{2}}$} \\
\hline \multicolumn{3}{|c|}{$\mathrm{RRC}^{1}$ of first lactation } & \multicolumn{3}{|c|}{ RRC of second lactation } & \multicolumn{3}{|c|}{ RRC of third lactation } & \\
\hline First & Second & Third & First & Second & Third & First & Second & Third & \\
\hline 3.735 & $\begin{array}{l}0.146 \\
0.398\end{array}$ & $\begin{array}{r}-0.479 \\
-0.262 \\
0.185\end{array}$ & $\begin{array}{l}0.837 \\
0.212 \\
0.447 \\
3.372\end{array}$ & $\begin{array}{r}-0.031 \\
0.697 \\
0.185 \\
0.059 \\
0.804\end{array}$ & $\begin{array}{r}-0.409 \\
-0.293 \\
0.909 \\
-0.438 \\
0.158 \\
0.254\end{array}$ & $\begin{array}{r}-0.840 \\
0.190 \\
-0.453 \\
0.970 \\
0.036 \\
-0.438 \\
3.712\end{array}$ & $\begin{array}{r}-0.042 \\
0.641 \\
0.101 \\
0.073 \\
0.962 \\
0.105 \\
0.067 \\
0.853\end{array}$ & $\begin{array}{r}-0.400 \\
-0.267 \\
0.909 \\
-0.433 \\
0.168 \\
0.977 \\
-0.430 \\
0.103 \\
0.294\end{array}$ & $\begin{array}{r}0.900 \\
0.199 \\
-0.403 \\
0.903 \\
-0.007 \\
-0.402 \\
0.903 \\
-0.006 \\
-0.403 \\
1.002\end{array}$ \\
\hline
\end{tabular}

${ }^{1} \mathrm{RRC}=$ random regression coefficient.

${ }^{2}$ France uses a single repeatability lactation model in national evaluation.

was higher than simulated ones, but this was due to the overparameterization of the model that disappears if the solution of one group is restricted to zero. When a year effect within country for each trait with a slope of $5 \%$ of the genetic standard deviation was simulated, the slope of the regression of the year effects estimated with the MT-MACE approach was very close to the simulated value $[0.0966(\mathrm{SE}=0.0074)$ vs. 0.0964 for the first RRC of the first lactation (Figure 2)]. Similar results were obtained for the year effects of the other traits. With the inclusion of this year effect, all estimated genetic correlations were similar to the simulated ones, on average. The standard deviations of these correlations were also small and similar to those of the reference situation. Therefore, it can be concluded that

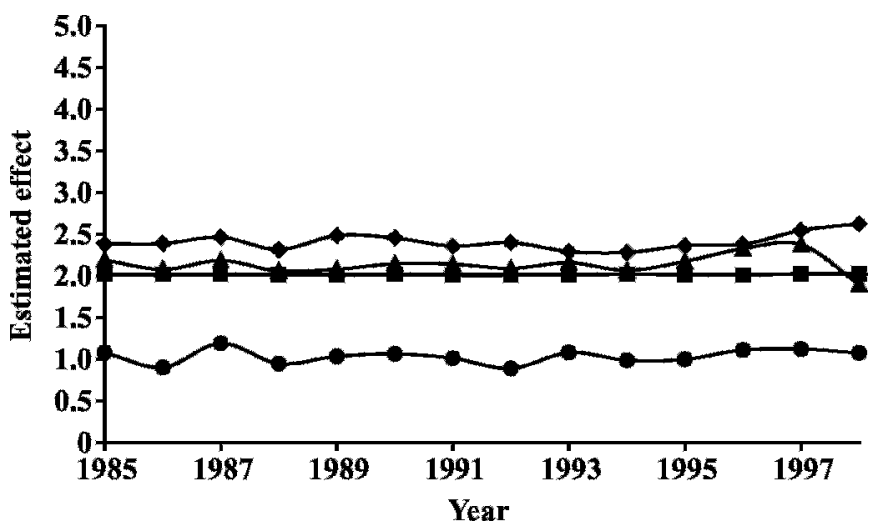

Figure 1. Estimated birth year effects under the multitrait, multiple across-country evaluation model for the German first random regression coefficient of the first lactation in 3 replicates $(\bullet, \bullet, \mathbf{\Delta})$ and average estimate over 20 replicates (ם) with no trend in simulated Mendelian sampling effect. including a time effect in international genetic evaluations will not alter the absence of bias for genetic parameters of the original MACE model. The inclusion of a time (birth year) effect in the joint evaluations can correct potential bias originated from national evaluations.

\section{DISCUSSION}

An MT-MACE model was proposed for international genetic evaluation to account for the fact that some countries use a multiple trait model in national genetic evaluation, whereas others have a single trait model. This MT-MACE model can better utilize the information derived from the RRTDM in national genetic evalu-

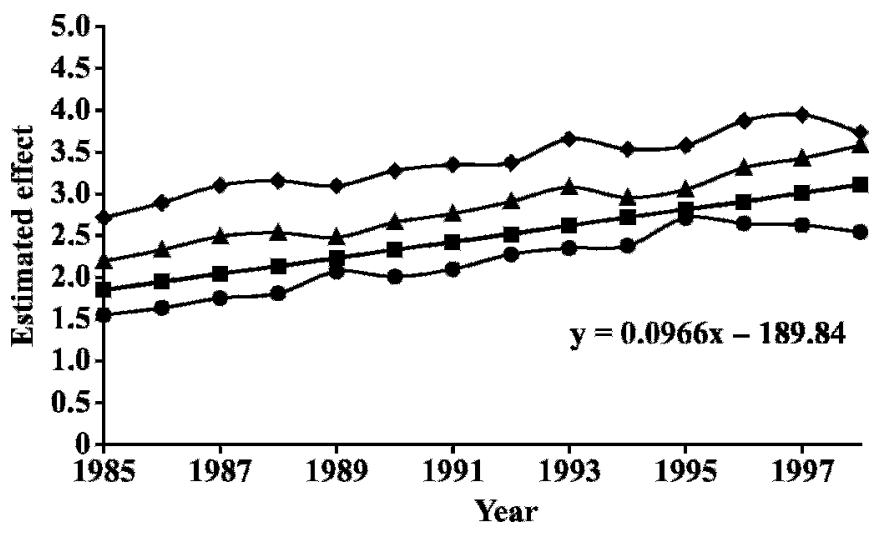

Figure 2. Estimated birth year effects under the multitrait, multiple across-country evaluation model for the German first random regression coefficient of the first lactation in 3 replicates $(\bullet, \bullet, \mathbf{\Delta})$ and average estimate over 20 replicates ( $\mathbf{\square}$ ) with simulated Mendelian sampling trend being $5 \%$ genetic standard deviations. 
ations than the ST-MACE model because it more closely models the additive genetic effects as in national evaluations. An approximate REML algorithm was developed for parameter estimation with this MT-MACE model. This algorithm avoids the inversion of the complete coefficient matrix of MME and approximates the inverse matrix via the MTEDC procedure. The results obtained in our simulation studies confirmed the suitability of the MT-MACE model and approximate EMREML method in a wide range of situations.

The approximate EM-REML gives nearly unbiased and precise estimates of across country genetic correlations. Note that REML does not give unbiased correlation estimates due to constraints on the parameter space. For example, suppose that the true correlation between Germany and France equals 1.0. In about half of simulated data sets, the estimated correlation would be less than 1.0 due to sampling error, and in the other half, the estimates would be 1.0, at the edge of the parameter space. The mean of the estimated correlation will show a downward bias. However, in our simulated study, the standard deviations of the estimates were small and this downward bias was less noticeable.

In the proposed MT-MACE model, only genetic (co)variances are to be estimated due to the assumption of zero residual covariances between countries. Error (co)variances within country do not need to be re-estimated either, because they are embedded (fixed) in EDC of bulls. The genetic (co)variances from individual countries can be used as starting values for the first iteration of parameter estimation to reduce time to convergence. However, even when starting values were far from country-specific values, we got nearly unbiased and precise estimates. We tried to fix national parameters ignoring the newly estimated country-specific (co)variances in step 4 of the procedure for parameter estimation and updating the covariances between countries using the correlation estimates and the original country parameters. The fixation of national parameters allowed a faster convergence because of the fewer parameters to be estimated. However, the estimated across country genetic correlations were biased. Further research should attempt to find a proper procedure to fix the national parameters.

A main advantage of the approximate EM-REML method is that large data sets can be analyzed; thus, the need for country subsetting and posterior bending currently practiced by Jorjani (2006) in the fertility implementation of MT-MACE can be reduced. The bending process to force the covariances to be positive definite leads to a general reduction of correlations (Jorjani, 2006). The size of the equation system and the need for country subsetting can also be reduced by extending to the MT-MACE model the factorial analysis approach developed by Leclerc et al. (2005) to reduce the number of estimated parameters. In fact, such an approach is even more important for the MT-MACE model with many more parameters than for the STMACE model.

Another question is of the inclusion of a year effect when using 2-step approaches for genetic evaluations. Currently, Interbull MACE evaluations only include a country mean as a fixed effect. However, predicted breeding values from national evaluations might be biased if selection over the past years is not considered, because the covariance structure of the traits is not taken into account. An approximate multitrait model, like MACE model, becomes robust to over- and underestimation of genetic parameters by adding a year effect in the model (Ducrocq et al., 2003; Lassen et al., 2007). The approximate EM-REML software was extended to fit an additional time effect; for example, birth year of animal, to integrate genetic trend validation into MACE evaluation. Including a year effect in the model gave correlations very close to the simulated ones. The year effect should be included in the joint analysis of the data to try to capture potential bias generated from national genetic evaluations. The inclusion of a fixed year effect to the model in place of the fixed country mean effect had an important impact on the top bull lists when a genetic trend existed.

In the recent past, different authors proposed MTMACE models based on de-regression approaches (Schaeffer, 2001; Sullivan et al., 2005; Mark and Sullivan, 2006) instead of a DYD-related approach. There are arguments for and against both approaches. In this study, the proposed MT-MACE model makes use of DYD of bulls together with corresponding EDC, but the MT-MACE software can integrate the de-regression step directly, because only the right hand side, $\Delta_{i j}$, of the MACE equation system needs to be obtained from the de-regression process. The current MT-MACE model can be applied to international cow evaluations, too. Because of the very high flexibility of the MT-MACE software, an application to cow evaluation does not require modification of source code. However, it is a challenging task to set up a reasonably complete and accurate international cow pedigree file. Finally, although the approximate EM-REML approach was proposed for parameter estimation before joint cow and bull international evaluation, it can also be applied to compute correlations between traits that are required for total merit index construction within country. In this case, the approximate REML has to be extended to estimate the error (co)variances because the assumption of zero residual correlations between traits is no longer valid, especially in cow evaluations. 


\section{CONCLUSIONS}

Based on the simulation studies, one can conclude that the MT-MACE model is a useful tool that can be implemented for joint cow and bull international evaluation. The developed software for parameter estimation based on an approximate EM-REML method works very well regarding the absence of bias and precision of parameter estimates. Fitting a time effect in the MACE model did not alter genetic parameters of the original MACE model. A year effect can be included in international evaluations to correct possible bias originating from national evaluations.

\section{ACKNOWLEDGMENTS}

The financial support by German Holstein Association (DHV) and Union Nationale des Coopératives d'Elevage et d'Insémination Animale (UNCEIA) of France is greatly appreciated.

\section{REFERENCES}

Druet, T., F. Jaffrezic, D. Boichard, and V. Ducrocq. 2003. Modeling lactation curves and estimation of genetic parameters for first lactation test-day records of French Holstein cows. J. Dairy Sci. 86:2480-2490.

Ducrocq, V., D. Boichard, A. Barbat, and H. Larroque. 2001. Implementation of an approximate multi-trait BLUP evaluation to combine production traits and functional traits into a total merit index. Page 2 in Proc. 52nd Annu. Mtg. Eur. Assoc. Anim. Prod., Budapest, Hungary. EAAP, Rome, Italy.

Ducrocq, V., I. Delaunay, D. Boichard, and S. Mattalia. 2003. A general approach for international genetic evaluations robust to inconsistencies of genetic trends in national evaluations. Interbull Bull. 30:101-111.

Fikse, W. F., L. Klei, Z. Liu, and P. G. Sullivan. 2003. Procedure for validation of trends in genetic variance. Interbull Bull. 31:30-35.

Jorjani, H. 2006. International genetic evaluation for female fertility traits. Interbull Bull. 34:57-60.

Lassen, J., M. K. Sorensen, P. Madsen, and V. Ducrocq. 2007. Robust models for approximate multitrait evaluations. Genet. Sel. Evol. 39:353-367.
Leclerc, H., W. F. Fikse, and V. Ducrocq. 2005. Principal components and factorial approaches for estimating genetic correlations in international sire evaluation. J. Dairy Sci. 88:3306-3315.

Liu, Z., F. Reinhardt, A. Bünger, L. Dopp, and R. Reents. 2001. Application of a random regression model to genetic evaluations of test day yields and somatic cell scores in dairy cattle. Interbull Bull. 27:159-166.

Liu, Z., F. Reinhardt, A. Bünger, and R. Reents. 2004a. Derivation and calculation of approximate reliabilities and daughter yield deviations of a random regression test-day model for genetic evaluation of dairy cattle. J. Dairy Sci. 87:1896-1907.

Liu, Z., F. Reinhardt, and R. Reents. 2004b. A multi-trait MACE model for international bull comparison based on daughter yield deviations. Interbull Bull. 32:46-52.

Mark, T., and P. G. Sullivan. 2006. Multiple-trait multiple-country genetic evaluations for udder health traits. J. Dairy Sci. 89:4874-4885.

Misztal, I., S. Tsuruta, T. Strabel, B. Auvray, T. Druet, and D. H. Lee. 2002. BLUF90 and related programs (BGF90). Proc. 7th World Congr. Genet. Appl. Livest. Prod., Montpellier, France. CD-ROM Commun. no. 28-07.

Mrode, R. A. 2005. Linear Models for the Prediction of Animal Breeding Values. 2nd ed. CAB International, Wallingford, UK.

Robert-Granié, C., B. Bonaiti, D. Boichard, and A. Barbat. 1999. Accounting for variance heterogeneity in French dairy cattle genetic evaluation. Livest. Prod. Sci. 62:343-357.

Schaeffer, L. R. 1994. Multiple-country comparison of dairy sires. J. Dairy Sci. 77:2671-2678.

Schaeffer, L. R. 2001. Multiple trait international bull comparison. Livest. Prod. Sci. 69:145-153.

Strandén, I., and M. Lidauer. 1999. Solving large mixed linear models using preconditioned conjugate gradient iteration. J. Dairy Sci. 82:2779-2787.

Sullivan, P., and J. W. Wilton. 2001. Multiple-trait MACE with a variable number of traits per country. Interbull Bull. 27:68-72.

Sullivan, P. G., J. W. Wilton, L. R. Schaeffer, G. J. Jansen, J. A. B. Robinson, and O. B. Allen. 2005. Genetic evaluation strategies for multiple traits and countries. Livest. Prod. Sci. 92:195-205.

Tarres, J., Z. Liu, V. Ducrocq, F. Reinhardt, and R. Reents. 2006. Parameter estimation and genetic evaluation of milk production traits from France and Germany with a multi-trait MACE model. Interbull Bull. 35:76-87.

VanRaden, P. M. 2001. Methods to combine estimated breeding values obtained from separate sources. J. Dairy Sci. 84(E Suppl.): E47-E55.

VanRaden, P. M., and G. R. Wiggans. 1991. Derivation, calculation, and use of national animal model information. J. Dairy Sci. 74:2737-2746. 